\title{
Evaluation of the response of breast cancer patients to neoadjuvant chemotherapy by combined contrast-enhanced ultrasonography and ultrasound elastography
}

\author{
BAOHUA WANG $^{1 *}$, TIAN'AN JIANG $^{1 *}$, MIN HUANG $^{1}$, JING WANG $^{1}$, \\ YANHUA CHU ${ }^{1}$, LIYUN ZHONG ${ }^{1}$ and SHUSEN ZHENG ${ }^{2}$ \\ ${ }^{1}$ Department of Ultrasound Medicine; ${ }^{2}$ Department of Surgery, Division of Hepatobiliary and Pancreatic Surgery, The \\ First Affiliated Hospital, Zhejiang University, Hangzhou, Zhejiang 310003, P.R. China
}

Received April 23, 2018; Accepted February 20, 2019

DOI: $10.3892 /$ etm.2019.7353

\begin{abstract}
The purpose of the present study was to investigate whether contrast-enhanced ultrasonography (CEUS) in combination with ultrasound elastography (UE) is able to accurately predict the efficacy of neoadjuvant chemotherapy (NAC) in breast cancer patients. A total of 65 breast cancer patients who received NAC at the First Affiliated Hospital of Zhejiang University (Hangzhou, China) between February 2016 and August 2017 and were recruited for the present study. Prior to and after NAC, examination by CEUS, UE or their combination was performed. Pathological results were obtained at the end of each chemotherapy cycle, based on which 41 cases were assigned to the response group and 24 to the non-response group. Kappa values were $0.710,0.434$ and 0.836 for CEUS, UE and CEUS+UE, respectively. The area under the receiver operating characteristic curves for CEUS, UE and CEUS+UE for determining the response to NAC was 0.864 [95\% confidence interval (CI), 0.765-0.964], 0.715 (95\% CI, 0.579-0.850) and 0.910 (95\% CI, 0.826-0.993), respectively. It was identified that the sensitivity, specificity, accuracy, positive predictive value and negative predictive value of CEUS+UE were higher than those of CEUS and US individually. The prediction accuracy was $89.2,90.8$ and $100 \%$ for CEUS, UE and their combination, respectively. CEUS and UE have their own advantages in evaluating the clinical efficacy of NAC in breast cancer, and a higher accuracy was achieved when the two techniques were applied in combination. Therefore, a combination of CEUS
\end{abstract}

Correspondence to: Dr Shusen Zheng, Department of Surgery, Division of Hepatobiliary and Pancreatic Surgery, The First Affiliated Hospital, Zhejiang University, 79 Qingchun Road, Shangcheng, Hangzhou, Zhejiang 310003, P.R. China

E-mail: shusenzheng@zju.edu.cn

*Contributed equally

Key words: contrast-enhanced ultrasonography, ultrasound elastography, neoadjuvant chemotherapy, breast cancer and UE may be a preferred method for the clinical assessment of the efficacy of NAC in breast cancer patients.

\section{Introduction}

Breast cancer, a malignant tumor type derived from the epithelium of terminal ductal lobules of the breast, is a disease that seriously threatens the health of women $(1,2)$. An estimated 1.2 million women suffer from breast cancer worldwide, and 500,000 women die from the disease each year $(3,4)$. In recent years, the incidence of breast cancer has increased in China $(5,6)$. The development of methods for the precise diagnosis, selection of a suitable treatment and prediction of the outcome, as well as studies on the mechanism of carcinogenesis, have become major research topics in the field of breast cancer $(7,8)$.

Neoadjuvant chemotherapy (NAC) is a systemic cytotoxic drug treatment method targeting the local malignant tumor prior to surgery and/or radiotherapy. NAC has now become an important component of comprehensive breast cancer treatment (9). While a large number of clinical trials have proved that NAC is effective in treating advanced breast cancer, studies have also indicated that $10-35 \%$ of patients were insensitive to chemotherapy drugs. The effect of chemotherapy is not significant in those patients, which allows the disease to progress (10-12). Therefore, the effectiveness of NAC should be more precisely evaluated in order to provide breast cancer patients with an effective treatment plan.

Breast ultrasound is a low-cost and non-invasive imaging method, and due to its advantages of high resolution and ability to visualize impalpable lumps, it is superior to mammography, computed tomography (CT) and magnetic resonance imaging (MRI) (13-17). With the popularization of ultrasound and the rapid development of novel ultrasonic technology, high-frequency ultrasound, elastography and ultrasound contrast have been frequently applied to patients with suspected breast cancer in the past years, which is helpful for improving the diagnostic accuracy (18-22). In the present study, contrast-enhanced ultrasonography (CEUS) and ultrasound elastography (UE) were used to evaluate the efficacy of NAC in breast cancer patients. 


\section{Patients and methods}

Patients. A total of 65 breast cancer patients who received NAC at the First Affiliated Hospital of Zhejiang University (Zhejiang, China) between February 2016 and August 2017 were recruited for the present study. All patients were confirmed via biopsy to have breast cancer prior to receiving NAC treatment and they received no other treatment. This study was approved by the ethics committee of the First Affiliated Hospital of Zhejiang University (Zhejiang, China). All patients provided written informed consent.

Chemotherapy regimen. Prior to surgery, all patients received 6 cycles of NAC with the 'TEC' chemotherapy regimen (docetaxel, $75 \mathrm{mg} / \mathrm{m}^{2}$; epirubicin, $75 \mathrm{mg} / \mathrm{m}^{2}$; cyclophosphamide, $500 \mathrm{mg} / \mathrm{m}^{2}$ ). Fluorescence in situ hybridization indicated that the tumors of 6 patients were positive for human epidermal growth factor receptor 2 , and they were given the targeted drug herceptin $(8 \mathrm{mg} / \mathrm{kg}$ body mass for the first time, followed by $6 \mathrm{mg} / \mathrm{kg}$ body mass). Drug treatment for 21 days was considered as 1 cycle and an interval of 20 days occurred following. Image examinations were performed prior to the next NAC cycle. Surgical excision was performed within 20 days after 6 cycles of drug treatment. A flow chart illustrating the chemotherapeutic regimen is presented in Fig. 1.

CEUS examination. CEUS was performed using an ESAOTE MyLabClassC ultrasound diagnostic instrument (Esaote SpA, Genoa, Spain). The ultrasound contrast agent SonoVue (59 $\mu \mathrm{g}$; Bracco SpA, Milan, Italy) was added to $5 \mathrm{ml}$ saline and a milky microbubble suspension was generated by vigorous agitation. The largest section of the nodule was identified and its location was entered into a real-time ultrasound contrast model (QontraXt, Version 3.06). A total of $4.8 \mathrm{ml}$ SonoVue suspension was rapidly injected through an anterior elbow vein and then $5 \mathrm{ml}$ of saline was injected to flush the tube. The dynamic image at $180 \mathrm{sec}$ was stored in real-time. Contrast observation continued until the lesion-enhanced image disappeared. The ultrasound contrast image was then processed, and the time-intensity curve was drawn for the region of interest (ROI). The parameters of CEUS including rise time (RT), time to peak (TTP), peak intensity (PI) and ascending slope (AS) were recorded. The differences in the parameters at the end of NAC vs. baseline $(\Delta)$ were calculated as follows: $\Delta \mathrm{AS}=(\mathrm{AS} 0-\mathrm{AS} 6) / \mathrm{AS} 0 \times 100 \%$ and $\Delta \mathrm{PI}=(\mathrm{PI} 0-\mathrm{PI} 6) / \mathrm{PI} 0 \times 100 \%$, where AS0 and AS6 or the PI0 and PI6 are the AS or PI at baseline and after the 6th cycle of NAC, respectively.

UE examination. For UE, the ESAOTE MyLabClass C ultrasound diagnostic instrument was used (Esaote SpA, Genoa, Spain). First, conventional ultrasound scanning was implemented to locate the breast nodules, followed by switching to elastic mode, in which breast elastography was performed. The number of the pressure indicators of the display screen remained between 2 and 3, and the ROI remained 2-3 times larger than the nodule. UE observation was dynamically performed. According to the '5 Points Grading Method' proposed by Itoh (23), the breast nodules were evaluated by elastic scoring, with 1-3 points indicating a benign, and 4 and 5 points suggesting a malignant status. The parameters of UE, including the strain ratio (SR) and strain point (SP), were recorded, and $\Delta$ values were calculated as follows: $\Delta \mathrm{SP}=(\mathrm{SP} 0-\mathrm{SP} 6) / \mathrm{SP} 0 \times 100 \% ; \Delta \mathrm{SR}=(\mathrm{SR} 0-\mathrm{SR} 6) / \mathrm{SR} 0 \times 100 \%$.

The differential diagnosis was established by two senior physicians based on the results of CEUS and UE. To assess whether the patient was responsive to NAC or not, the sensitivity, specificity and accuracy of CEUS, UE and the combination of the two were analyzed by comparison with the post-operative pathological diagnosis, which was considered as the gold standard.

CEUS and UE evaluation. According to the response evaluation criteria in solid tumors (24), changes in the longest diameter of the lesion prior to and after chemotherapy were compared. A total reduction of the maximum diameter of the lesion of $\geq 30 \%$, or disappearance of the lesion with no new lesion appearing, was defined as a 'response' to NAC. A 'non-response' was defined as the longest diameter of the original lesion being decreased by $<30 \%$ or increased after NAC, or the appearance of a new focus.

Pathological evaluation. According to the tumor response grade proposed by Eisenhauer et al (25), the histopathology results of the lesion samples isolated in the surgery were compared with those of the biopsy specimens obtained prior to treatment to determine the response to NAC, based on which the patients were stratified into two groups: A reduction in the relative number of cancer cells by $<30 \%$ was defined as 'non-response', while a 'response' was considered when cancer cells were reduced by $\geq 30 \%$.

Statistical analysis. The data were analyzed by SPSS version 20 statistics software (IBM Corp., Armonk, NY, USA). The Chi square test was used for evaluation of count data. The Kappa test was performed to analyze the results of CEUS and UE to evaluate the outcome of NAC as well as the consistency with the results of the post-operative pathological evaluation. Parameter changes of CEUS and UE were assessed by Student's t-test. The prediction rate was calculated using univariate logistic regression analysis. Receiver operating characteristic (ROC) curves and the area under the curve (AUC) were obtained for CEUS and UE to evaluate NAC efficacy. P $<0.05$ was considered to indicate a statistically significant difference.

\section{Results}

Characteristics of the patients and their tumors. The baseline characteristics of the patients are presented in Table I. No significant differences in the clinicopathological characteristics at baseline were identified between the response and no response groups. Post-operative histopathological analysis indicated that the density of cancers cells was relatively high in the tumors from non-response patients, while it was relatively low in the response patients (Fig. 2A and B). Invasive ductal carcinoma of the breast was confirmed through tissue coarse needle biopsy prior to NAC (Fig. 2C). After NAC, the surgical specimen was confirmed as grade 4 (Fig. 2D).

Performance of CEUS and UE in evaluating the efficiency of $N A C$. The Kappa test was used to analyze the consistency of 


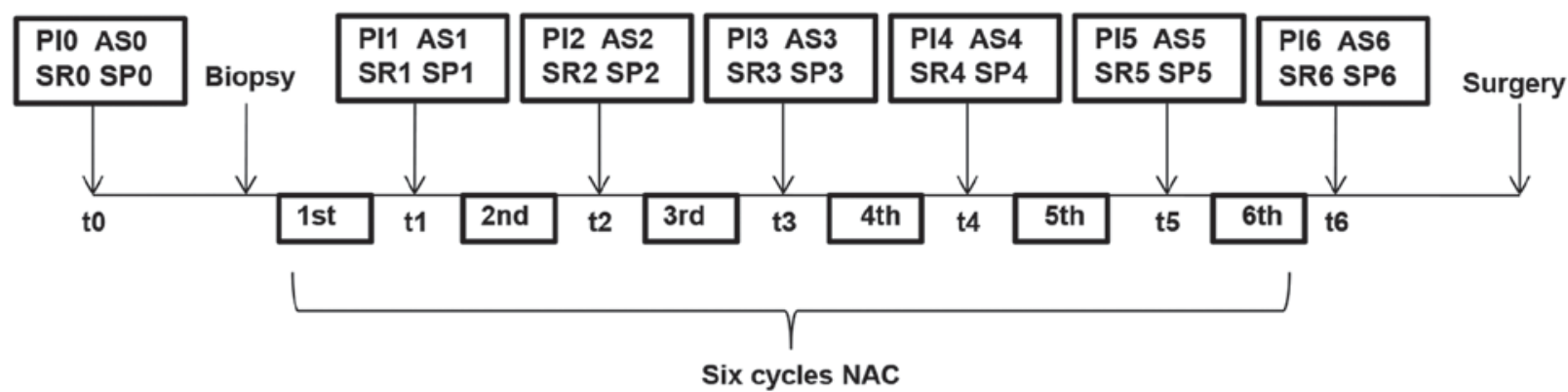

Figure 1. Flow chart of NAC. Each cycle lasted for 21 days and surgical excision was performed within 20 days after 6 cycles. The parameters of CEUS and UE were recorded, including PI, AS, SR and SP. NAC, neoadjuvant chemotherapy; PI, peak intensity; AS, ascending slope; SR, strain ratio; SP, strain pressure; CEUS, contrast-enhanced ultrasonography; UE, ultrasound elastography.

Table I. Clinicopathological features of the patients at baseline.

\begin{tabular}{lccc}
\hline Characteristic & $\begin{array}{c}\text { Response } \\
\text { group (n=41) }\end{array}$ & $\begin{array}{c}\text { No response } \\
\text { group (n=24) }\end{array}$ & P-value \\
\hline Age (years) & $48.3 \pm 5.7$ & $50.2 \pm 4.9$ & 0.170 \\
$\begin{array}{l}\text { Menopausal status } \\
\text { Pre-menopause }\end{array}$ & $21(51.21 \%)$ & $10(41.67 \%)$ & 0.608 \\
Post-menopause & $20(18.79 \%)$ & $14(58.33 \%)$ & \\
Tumor size (cm) & & & 1.000 \\
$>4$ & $10(24.39 \%)$ & $5(20.83 \%)$ & \\
$<4$ & $31(75.61 \%)$ & $19(79.17 \%)$ & \\
Histological grade & & & 1.000 \\
2 & $25(60.98 \%)$ & $15(62.50 \%)$ & \\
3 & $16(39.02 \%)$ & $9(37.50 \%)$ & \\
Tumor subtype & & & 0.601 \\
Luminal A & $14(34.15 \%)$ & $10(41.67 \%)$ & \\
Luminal B & $27(65.85 \%)$ & $14(58.33 \%)$ & \\
Nodal status & & & 0.785 \\
Negative & $29(70.73 \%)$ & $16(66.67 \%)$ & \\
Positive & $12(29.27 \%)$ & $8(33.33 \%)$ & \\
\hline
\end{tabular}

Values are expressed as the mean \pm standard deviation or $\mathrm{n}(\%)$.

the evaluation results of CEUS, US and their combination with the results of the post-operative pathological evaluation. The pathological results indicated that 41 cases exhibited a response to NAC and 24 cases did not (Table II). A Kappa value of $>0.75$ indicated good consistency, $0.75>$ Kappa $>0.4$ suggested moderate consistency and Kappa $<0.4$ was considered to indicate poor consistency. This suggested that combination of CEUS and UE have a good efficiency in predicting the response to NAS based on histopathological examinations. CEUS was also demonstrated to do this more efficiently than UE. In addition, the sensitivity, specificity, accuracy, positive predictive value (PPV) and negative predictive value (NPV) of CEUS and UE were analyzed. The AUC of the ROC for the prediction of a response by CEUS was 0.864 [95\% confidence interval (CI), 0.765-0.964]. Furthermore, the AUC for UE was 0.715 (95\% CI, 0.579-0.850) and that of CEUS+UE was 0.910 (95\% CI, 0.826-0.0.993; Fig. 3). The sensitivity, specificity, accuracy, PPV and NPV of CUES and of UE are presented in
Table III. It was observed that the AUC, sensitivity, specificity, accuracy and PPV of CEUS+UE were higher than those of CEUS or UE alone.

Image and parameter analysis of CEUS and UE. The CEUS images were analyzed using Philips QLAB ultrasound contrast quantitative analysis software (Fig. 4). The maximum signal intensity in the non-response group was identified to be stronger than that in the response group (Fig. 4). The corresponding time-signal intensity curve in the response group was more stable than that in the non-response group (Fig. 4B and D). Subsequently, the parameters of CEUS obtained from the images were analyzed. In comparison with those in the non-response group, the PI and ascending slope AS were significantly reduced in the response group, while the RT and TTP remained relatively stable. Furthermore, it was identified that compared with the baseline values, the PI and AS were obviously decreased after NAC in the non-response and response groups (Table IV).

CEUS and UE imaging was performed prior to the next NAC cycle, and prior to the operation. The images revealed that compared with the non-response group, the residual tumor in the response group was markedly reduced (Fig. 5). Subsequently, the parameters of UE were determined based on the images. The results indicated that the percentage of cases with 1-3 points according to the ' 5 Points Grading Method' in the response group was higher than that in the non-response group. However, the percentage of cases with 4 or 5 points in the response group was lower than that in the non-response group (Table V). Furthermore, the strain ratio (SR) after NAC was conspicuously reduced in the response group compared with that the non-response group $(2.11 \pm 0.52$ vs. $3.71 \pm 1.29)$, whereas no differences were observed prior to NAC between them. In addition, in comparison with the baseline, the number of the cases with 1-3 points was significantly increased, while the SR and the number of the cases with 4 and 5 points were markedly decreased in either group after NAC (Table V).

Accuracy of CEUS and UE in predicting the efficiency of $N A C$. ROC curves, the optimal cutoff and the area under the ROC (AUC) were obtained for the parameters of CEUS and UE, namely $\Delta \mathrm{AS}, \Delta \mathrm{PI}, \Delta \mathrm{SP}$ and $\Delta \mathrm{SR}$ (Fig. 6). The optimal cutoff values for $\Delta \mathrm{AS}, \Delta \mathrm{PI}, \Delta \mathrm{SP}$ and $\Delta \mathrm{SR}$ were $>36.94,21.15$, 40 and $52.50 \%$, respectively. ROC curve analysis for $\triangle \mathrm{AS}$ indicated that the sensitivity, specificity, accuracy, PPV and 
Table II. Comparison of evaluation of CEUS and UE with pathological evaluation.

\begin{tabular}{|c|c|c|c|c|c|}
\hline \multirow[b]{2}{*}{ Imaging modality/result } & \multirow[b]{2}{*}{ Total n (\%) } & \multicolumn{2}{|c|}{ Histology } & \multirow[b]{2}{*}{ Карра } & \multirow[b]{2}{*}{ P-value } \\
\hline & & Response & No response & & \\
\hline \multicolumn{6}{|l|}{ CEUS } \\
\hline Response & $38(58.46 \%)$ & $35(85.37 \%)$ & $3(14.29 \%)$ & 0.710 & $<0.001$ \\
\hline No response & $27(41.54 \%)$ & $6(14.63 \%)$ & $21(85.71 \%)$ & & \\
\hline Total & 65 & 41 & 24 & & \\
\hline \multicolumn{6}{|l|}{ UE } \\
\hline Response & $42(64.62 \%)$ & $33(80.49 \%)$ & $9(37.50 \%)$ & 0.434 & $<0.001$ \\
\hline No response & $23(35.38 \%)$ & $8(19.51 \%)$ & $15(62.50 \%)$ & & \\
\hline Total & 65 & 41 & 24 & & \\
\hline \multicolumn{6}{|l|}{ CEUS+UE } \\
\hline Response & $40(61.54 \%)$ & $38(92.68 \%)$ & $2(4.88 \%)$ & 0.836 & $<0.001$ \\
\hline No response & $25(38.45 \%)$ & $3(7.32 \%)$ & $22(95.12 \%)$ & & \\
\hline Total & 65 & 41 & 24 & & \\
\hline
\end{tabular}

CEUS, contrast-enhanced ultrasonography; UE, ultrasound elastography.

A

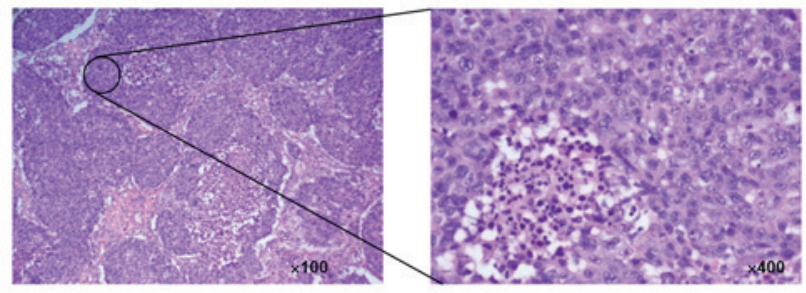

B

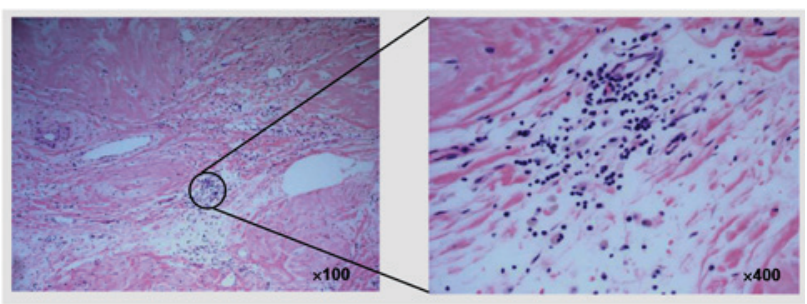

C

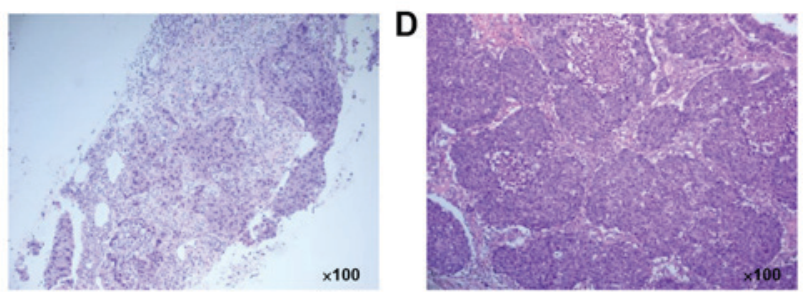

Figure 2. Histopathological identification of patient responses with $\mathrm{H} \& \mathrm{E}$ staining. Representative H\&E staining of histology images of patients in (A) the non-response group and (B) the response group (magnification in left panel, $x 100$; the right panel shows the windows from the left panel at a magnification of x400). (C) Representative H\&E staining of tissue coarse needle biopsy from one patient in the response group was performed and invasive ductal carcinoma of the breast was confirmed prior to NAC. (D) Following NAC, H\&E staining of the surgical specimen of this patient was confirmed as grade 4 (magnification, x100). NAC, neoadjuvant chemotherapy.

NPV were $87.80,95.83,90.76,97.29$ and $82.14 \%$, respectively, while they were $90.24,95.83,92.30,97.37$ and $85.18 \%$ for $\Delta$ PI. Furthermore, the sensitivity, specificity, accuracy, PPV

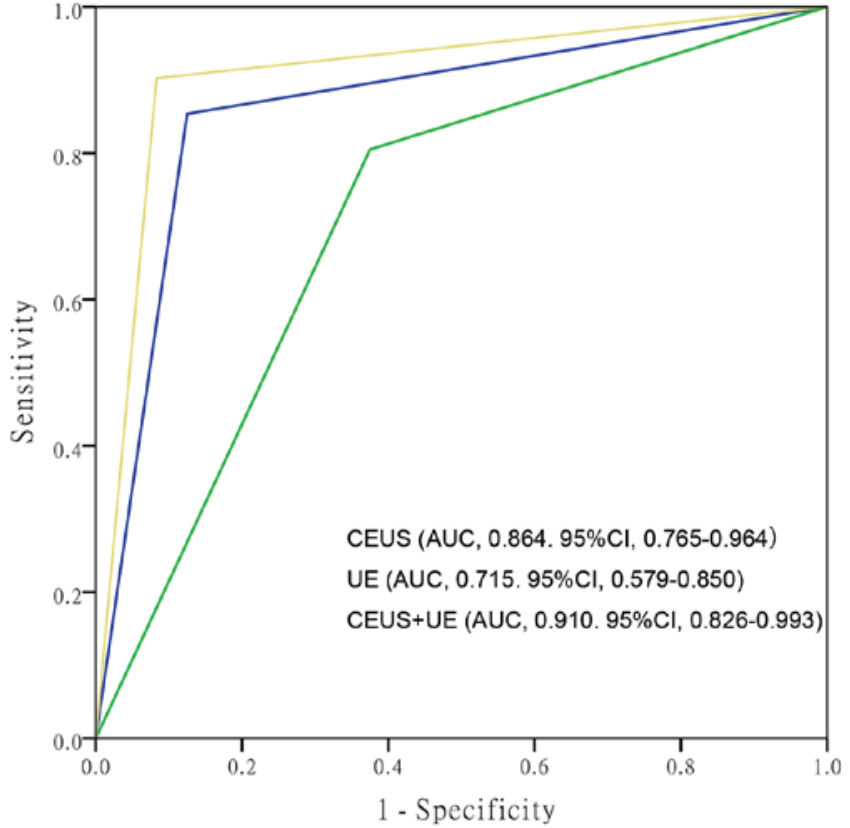

Figure 3. ROC analysis for CEUS, UE and their combination in the prediction of the response to NAC in breast cancer patients. $\mathrm{CI}$, confidence interval; AUC, area under the ROC curve; ROC, receiver operating characteristic; CEUS, contrast-enhanced ultrasonography; UE, ultrasound elastography.

and NPV for $\Delta$ SP and $\Delta$ SR were 65.85 and $92.68 \%, 83.33$ and $87.50 \%, 72.30$ and $90.76 \%, 87.09$ and $92.68 \%$, and 58.82 and $87.50 \%$, respectively (Table VI). The prediction accuracy rates of CEUS, UE and CEUS+UE were determined by a combination of $\Delta \mathrm{PI}, \Delta \mathrm{AS}, \Delta \mathrm{SP}$ or $\Delta \mathrm{SR}$ using logistics analysis (Table VII). The CEUS prediction rate with $\Delta \mathrm{PI}$ and $\Delta \mathrm{AS}$ as the influence factors was $89.2 \%$, whereas the UE prediction rate with $\Delta \mathrm{SP}$ and $\Delta \mathrm{SR}$ as the influence factors was $90.8 \%$. The combination prediction rate of CEUS+UE with $\Delta \mathrm{SP}, \Delta \mathrm{SR}$ and $\Delta \mathrm{PI}$ as the influence factors was $100 \%$. 
Table III. Comparison of sensitivity, specificity, accuracy, PPV and NPV (\%) between CEUS, UE and their combination.

\begin{tabular}{lccc}
\hline Parameter & CEUS & UE & CEUS+UE \\
\hline Sensitivity & 85.36 & 80.49 & 92.68 \\
Specificity & 87.50 & 62.50 & 91.67 \\
Accuracy & 86.15 & 73.85 & 92.31 \\
PPV & 92.11 & 78.57 & 95.00 \\
NPV & 77.77 & 65.22 & 87.99 \\
\hline
\end{tabular}

PPV, positive predictive value; NPV, negative predictive value; CEUS, contrast-enhanced ultrasonography; UE, ultrasound elastography.

A

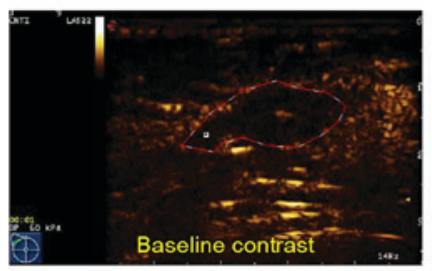

C

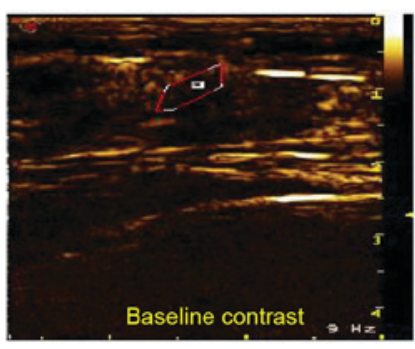

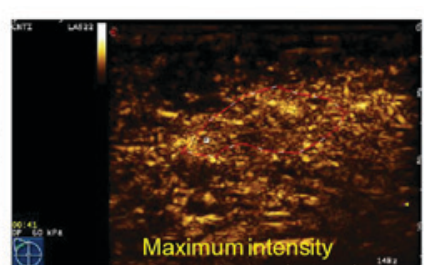

B

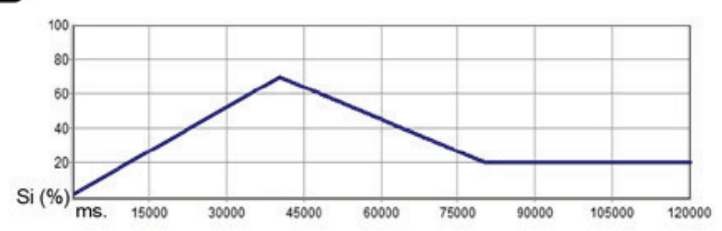

D

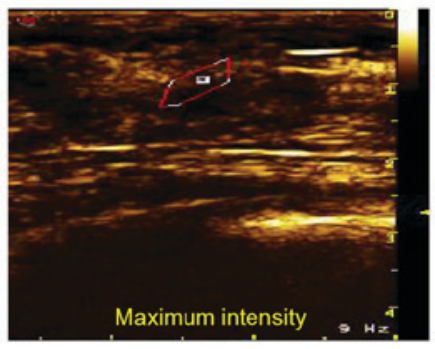

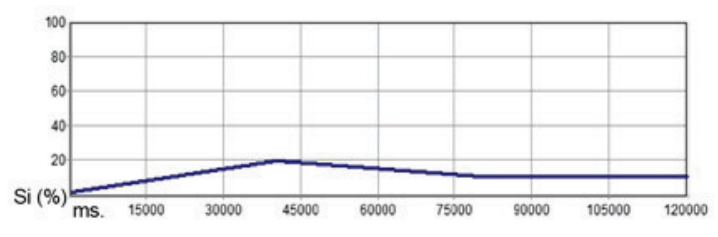

Figure 4. Representative CEUS images of non-response/response patients. (A) CEUS image and (B) time-Si curve for a non-response patient. (C) CEUS image and (D) time-Si curve for a patient with response to neo-adjuvant chemotherapy. Areas gated with red lines represent the region of interest. CEUS, contrast-enhanced ultrasonography; $\mathrm{Si}$, signal intensity; ms, milliseconds.

\section{Discussion}

Since the 1970s, NAC has been a critical part of the comprehensive treatment of breast cancer. NAC may reduce tumor cells and significantly increase the rate of breast-conserving and surgical resection $(26,27)$. Pathological examination is a gold standard for evaluating tumor response after a patient received NAC and it has a high diagnostic accuracy. However, in addition to the post-operative examination, the efficiency of NAC should ideally be determined at the end of/during NAC, as the pre-operative prediction of the pathological response to NAC allows for individualized breast cancer surgery timing $(28,29)$. Therefore, recent studies have encouraged the use of repeated, non-invasive monitoring by imaging examinations so as to evaluate the efficacy of NAC $(17,30,31)$.

Imaging evaluation of the response of breast cancer to $\mathrm{NAC}$ is required to be performed from two aspects of qualitative and quantitative data. While a unified assessment system is currently lacking, the evaluation standard regarding the size of the lesion is unified. The standard is to compare the longest diameter in images prior to and after NAC and to thereby evaluate the response. After NAC, the regression of tumor cells mainly manifests as necrosis and fibrosis of the lesions $(32,33)$. Conventional ultrasound cannot accurately distinguish between necrosis, fibrous hyperplasia and residual cancer (34).

Breast cancer is a vasculature-derived disease and with abundant neovascularization. The formation of neovascularization is critically associated with the prognosis of patients with breast cancer $(35,36)$. CEUS is able to detect microvessels of $<40 \mu \mathrm{m}$ in diameter and to clearly display the shape and distribution of the lesion, as well as the blood vessels $(31,37)$. CEUS has proven effective in evaluating the clinical response of various cancer types to NAC $(14,30,38,39)$. In the present study, CEUS was identified to have a good performance in evaluating the effect of NAC. The parameters of PI and AS were significantly decreased in the responsive patients compared to those in the patients that were unresponsive to NAC. Furthermore, in comparison with the baseline, the PI and AS were also markedly reduced after NAC in the response and non-response groups after NAC. CEUS predicted the effectiveness of NAC with the rate of correct prediction of the response to NAC being $89.2 \%$. The above results proved that CEUS may be used to efficiently predict the pathological response of breast tumors to NAC.

$\mathrm{UE}$ is able to reflect the elastic characteristics of various biological tissues, and may provide useful clues for diagnosing 
Table IV. Quantitative parameters of CEUS prior to and after NAC.

\begin{tabular}{|c|c|c|c|}
\hline Parameter/time-point & Response $(n=41)$ & No response $(n=24)$ & P-value \\
\hline RT after NAC & $9.43 \pm 2.61$ & $8.25 \pm 2.67$ & 0.086 \\
\hline TTP after NAC & $19.05 \pm 4.17$ & $21.10 \pm 4.37$ & 0.065 \\
\hline \multicolumn{4}{|l|}{ PI } \\
\hline Prior to NAC & $26.18 \pm 3.82$ & $25.32 \pm 4.11$ & 0.398 \\
\hline After NAC & $13.87 \pm 3.77^{\mathrm{a}}$ & $22.34 \pm 4.05^{\mathrm{b}}$ & $<0.001$ \\
\hline \multicolumn{4}{|l|}{ AS } \\
\hline Prior to NAC & $1.65 \pm 0.50$ & $1.41 \pm 0.42$ & 0.052 \\
\hline After NAC & $0.78 \pm 0.29^{\mathrm{a}}$ & $1.06 \pm 0.24^{\mathrm{a}}$ & $<0.001$ \\
\hline
\end{tabular}

${ }^{\mathrm{a}} \mathrm{P} \leq 0.001 ;{ }^{\mathrm{b}} \mathrm{P}<0.05$ vs. prior to NAC. CEUS, contrast-enhanced ultrasonography; NAC, neoadjuvant chemotherapy; PI, peak intensity; TTP, time to peak; PI, peak intensity; AS, ascending slope.

A

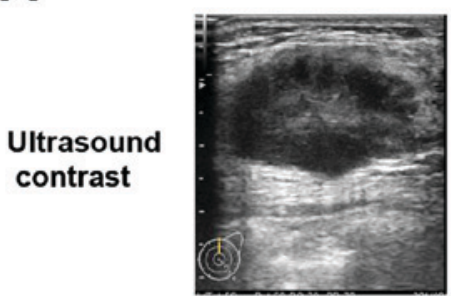

Pre-Tr

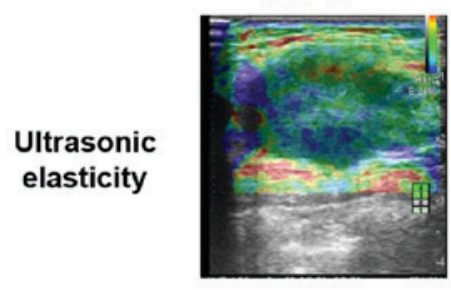

B

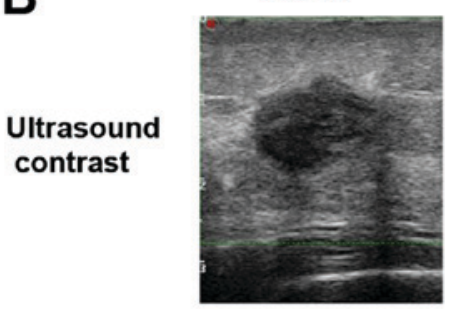

Pre-Tr

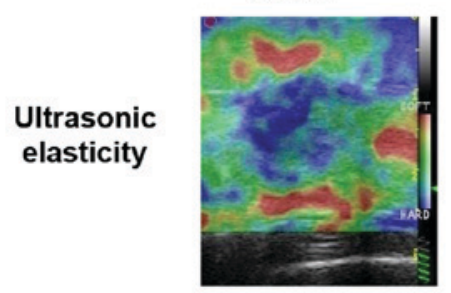

W1

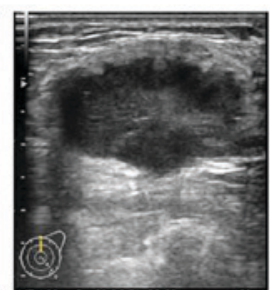

W1

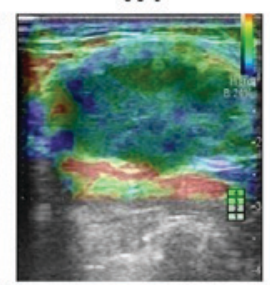

W1

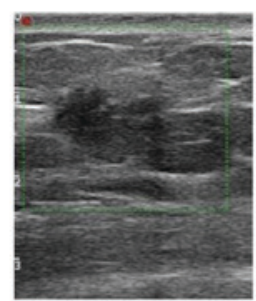

W1

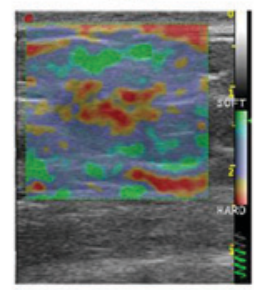

W4

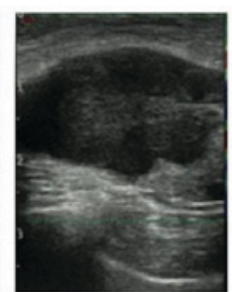

W4

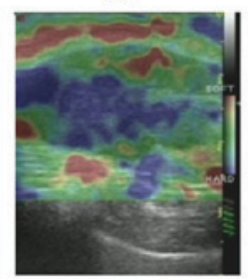

W4

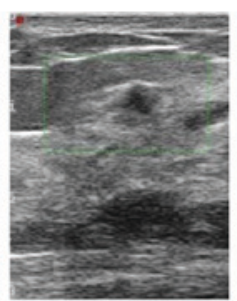

W4

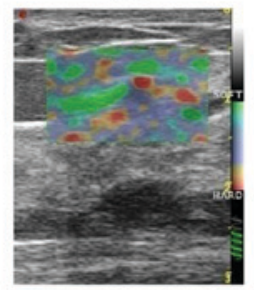

W8

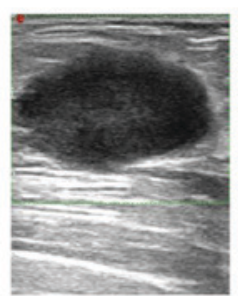

W8

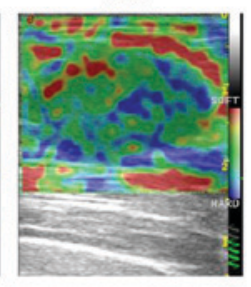

W8

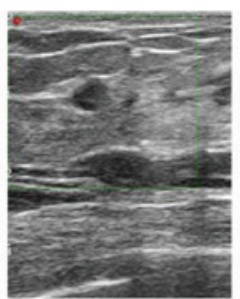

W8

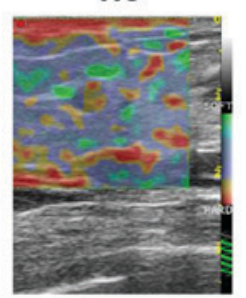

Pre-Op

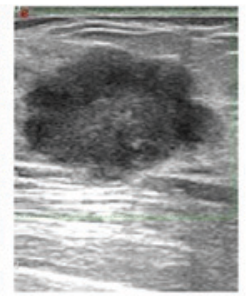

Pre-Op

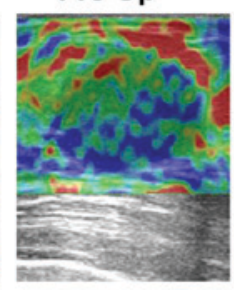

\section{Pre-Op}

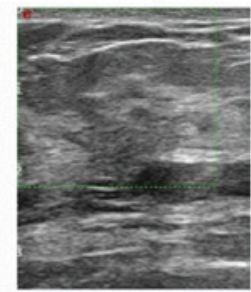

Pre-Op

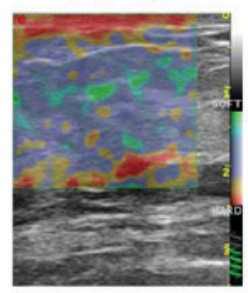

Figure 5. Representative images of CEUS and UE. CEUS and UE images of (A) a non-response patient and (B) a response patient obtained prior to the next NAC cycle and during the pre-operative diagnosis. CEUS, contrast-enhanced ultrasonography; UE, ultrasound elastography; W8, week 8; Pre-tr, pre-treatment; Pre-Op, pre-operative.

breast diseases; therefore, it is regarded as a beneficial supplementary method to conventional ultrasound (19). The most commonly used UE techniques are elastic scoring and the SR method $(40,41)$. Previous studies have indicated that by 
Table V. Comparison of points of UE evaluated using the '5 Point Grading Method' (23) prior to and following NAC.

\begin{tabular}{lccccccc}
\hline & \multicolumn{3}{c}{ Response $(\mathrm{n}=41)$} & & \multicolumn{3}{c}{ No response $(\mathrm{n}=24)$} \\
\cline { 2 - 4 } Time-point & $1-3$ points & $4 / 5$ points & SR & & $1-3$ points & $4 / 5$ points & SR \\
\hline Prior to NAC & 10 & 31 & $6.70 \pm 1.46$ & & 5 & 19 & $5.89 \pm 1.33$ \\
After NAC & 34 & 7 & $2.11 \pm 0.52$ & & 14 & 10 & $3.71 \pm 1.29^{\text {a }}$ \\
P-value & $<0.001$ & & $<0.001$ & & 0.008 & & $<0.001$
\end{tabular}

UE, ultrasound elastography; NAC, neoadjuvant chemotherapy; SR, strain ratio; ${ }^{a} \mathrm{P}<0.0001$ vs. the response for SR.

Table VI. Sensitivity, specificity, accuracy, PPV and NPV of CEUS and UE in the prediction of response to neoadjuvant chemotherapy $(\%)$.

\begin{tabular}{llllll}
\hline & \multicolumn{2}{c}{ CEUS } & & \multicolumn{2}{c}{ UE } \\
\cline { 2 - 3 } \cline { 6 - 6 } Parameter & $\Delta \mathrm{AS}$ & $\Delta \mathrm{PI}$ & & $\Delta \mathrm{SP}$ & $\Delta \mathrm{SR}$ \\
\hline Sensitivity & 87.80 & 90.24 & & 65.85 & 92.68 \\
Specificity & 95.83 & 95.83 & & 83.33 & 87.50 \\
Accuracy & 90.76 & 92.30 & & 72.30 & 90.76 \\
PPV & 97.29 & 97.37 & & 87.09 & 92.68 \\
NPV & 82.14 & 85.18 & & 58.82 & 87.50 \\
\hline
\end{tabular}

PPV, positive predictive value; NPV, negative predictive value; CEUS, contrast-enhanced ultrasonography; UE, ultrasound elastography; $\Delta$, difference between baseline and 6 cycles of NAC; AS, ascending slope; PI, peak intensity; SP, strain point; SR, strain ratio.

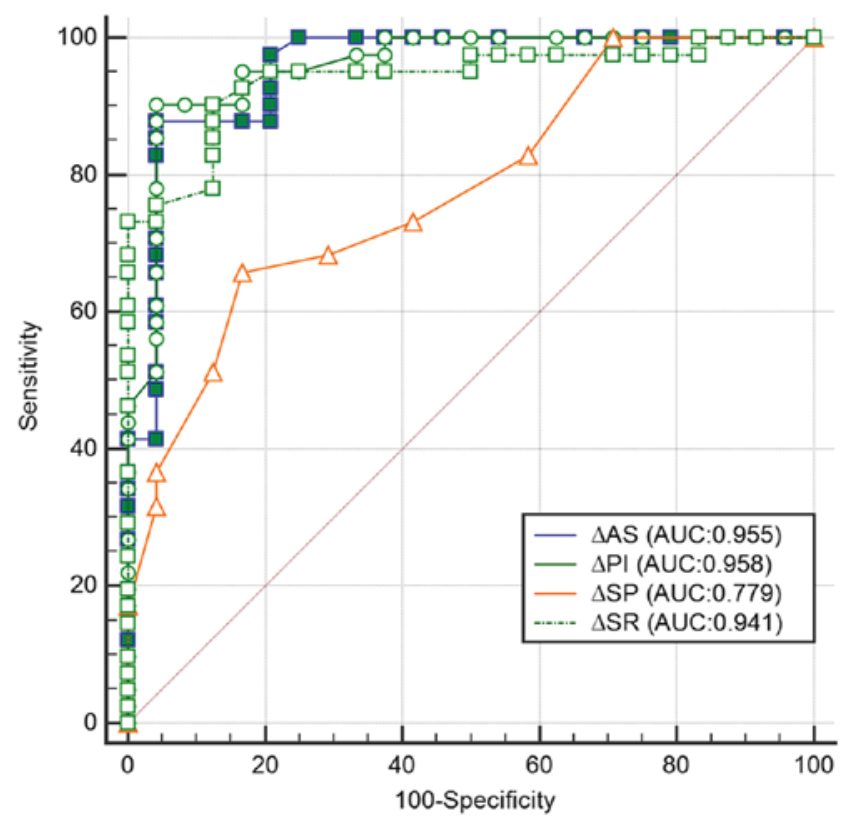

Figure 6. Analysis of the ROC curves of CEUS and UE in the prediction of the response to NAC in breast cancer patients. The receiver operating characteristics curves of $\Delta \mathrm{AS}, \Delta \mathrm{PI}, \Delta \mathrm{SP}$ and $\Delta \mathrm{SR}$ for prediction of the response to NAC. AUC, area under the ROC curve; ROC, receiver operating characteristic; $\Delta$, difference between baseline and 6 cycles of NAC; AS, ascending slope; PI, peak intensity; SP, strain point; SR, strain ratio; NAC, neoadjuvant chemotherapy.
Table VII. Prediction rate of CEUS and UE according to univariate logistic regression analysis ${ }^{\mathrm{a}}$.

\begin{tabular}{lccc}
\hline Modality/parameter & $\beta$ & P-value & OR (95\% CI) \\
\hline CEUS & & & \\
$\Delta \mathrm{PI}$ & -0.102 & 0.084 & $0.903(0.804-1.014)$ \\
$\Delta \mathrm{AS}$ & -0.094 & 0.179 & $0.910(0.793-1.044)$ \\
Constant & 8.636 & 0.001 & - \\
UE & & & \\
$\Delta$ SP & 0.067 & 0.010 & $1.069(1.016-1.125)$ \\
$\Delta$ SR & 0.144 & $<0.001$ & $1.155(1.074-1.242)$ \\
Constant & -9.964 & $<0.001$ & - \\
CEUS+UE & & & \\
$\Delta$ SP & 0.786 & 0.996 & 2.194 \\
$\Delta$ SR & 1.218 & 0.993 & 3.380 \\
$\Delta$ PI & -2.058 & 0.993 & 0.128 \\
Constant & -12.628 & 0.999 & - \\
\hline
\end{tabular}

The P-value of $\Delta \mathrm{AS}(0.179)$ was $>0.1$ and as such was not included in the combination analysis (CEUS+UE). CEUS, contrast-enhanced ultrasonography; UE, ultrasound elastography; $\Delta$, difference between baseline and 6 cycles of NAC; AS, ascending slope; PI, peak intensity; SP, strain point; SR, strain ratio; PI, peak intensity; CI, confidence interval; OR, odds ratio; constant, constant of the logistic regression equation. ${ }^{a}$ Logistics regression model: $\ln \frac{P}{1-P}=\beta 0+\beta 1 X 1+\cdots \beta \mathrm{nXn}$ (for CEUS, $\beta 0$ : Constant; $\Delta$ PI: $\beta 1 ; \Delta$ AS: $\beta 2$. for UE, $\beta 0$ : Constant; $\Delta$ SP: $\beta 1 ; \Delta \mathrm{SR}: \beta 2$. for CEUS+UE, $\beta 0$ : Constant; $\Delta \mathrm{SP}: \beta 1 ; \Delta \mathrm{SR}: \beta 2 ; \Delta \mathrm{PI}: \beta 3$ ).

combination of the two-dimensional grayscale image and the elastic scoring method, a high accuracy in the diagnosis of benign and malignant breast nodules was achieved (40). Hence, in the present study, the methods of elastic scoring and SR were also selected so as to explore the aptness of UE for evaluation of the efficacy of NAC. The rate of correct prediction of the response to NAC of UE with $\triangle \mathrm{SP}$ and $\triangle \mathrm{SR}$ as influence factors was $90.8 \%$. These results suggested that UE may be used to assess the pathological response of breast tumors to NAC.

To the best of our knowledge, no previous study has evaluated the combined use of CEUS and UE for evaluation of the efficacy of NAC for breast cancer. It was hypothesized that CEUS in combination with UE may predict NAC more accurately than CEUS alone. As expected, the predictive accuracy of the combined evaluation by CEUS or UE was higher than that of each imaging modality implemented individually. 
When CEUS was combined with UE, the sensitivity, specificity, accuracy, PPV and NPV were identified to be higher than those of CEUS or UE applied individually. Furthermore, the predictive accuracy rate of combined CEUS and UE was $100 \%$, proving that CEUS in combination with UE was highly accurate in evaluating the efficacy of NAC for breast cancer.

The present clinical study demonstrated that CEUS and UE have their individual advantages in evaluating the clinical efficacy of NAC for breast cancer, and that the combination of the two was more accurate. It is therefore recommended that this combined method is widely implemented in the clinical assessment of the efficacy of NAC for breast cancer patients. The present study is of great clinical significance and paves the road for further in-depth research on the combined use of CEUS and UE so as to predict the pathological response of breast cancer after patients have received NAC.

\section{Acknowledgements}

Not applicable.

\section{Funding}

The current study was supported by the Education Agency of Zhejiang Province (grant no. Y201534607), the Health Bureau of Zhejiang Province (grant no. 2017195594) and the Youth Talent Project of the Zhejiang Province Medical and Health Program (grant no. 2016148397).

\section{Availability of data and materials}

The data sets generated and analyzed during the study are available from the corresponding author on reasonable request.

\section{Authors' contributions}

BW, $\mathrm{MH}$ and JW were responsible for the collection and screening of cases. BW, TJ, YC, LZ and SZ performed the statistical analysis and analyzed the data. BW, TJ and JW wrote and revised the manuscript. The final version of the manuscript has been read and approved by all authors, and each author believes that the manuscript represents honest work.

\section{Ethical approval and consent to participate}

This study was approved by the ethics committee of the First Affiliated Hospital of Zhejiang University (Zhejiang, China). All patients provided written informed consent.

\section{Patient consent for publication}

Not applicable.

\section{Competing interests}

The authors declare that they have no competing interests.

\section{References}

1. Calado A, Neves PM, Santos T and Ravasco P: The effect of flaxseed in breast cancer: A literature review. Front Nutr 5: 4, 2018.
2. Nakayama T, Sagara Y, Takashima T, Matsunami N, Masuda N, Miyoshi Y, Taguchi T, Aono T, Ito T, Kagimura T and Noguchi S: Randomized phase II study of anastrozole plus tegafur-uracil as neoadjuvant therapy for ER-positive breast cancer in postmenopausal Japanese women (Neo-ACET BC). Cancer Chemother Pharmacol 81: 755-762, 2018.

3. Oeffinger KC, Fontham ET, Etzioni R, Herzig A, Michaelson JS, Shih YC, Walter LC, Church TR, Flowers CR, LaMonte SJ, et al: Breast cancer screening for women at average risk: 2015 guideline update from the American Cancer Society. JAMA 314: 1599-1614, 2015.

4. Vidula N and Rugo HS: Cyclin-dependent kinase 4/6 inhibitors for the treatment of breast cancer: A review of preclinical and clinical data. Clin Breast Cancer 16: 8-17, 2016.

5. Guo C, Sung H, Zheng S, Guida J, Li E, Li J, Hu N, Deng J, Figueroa JD, Sherman ME, et al: Age-related terminal duct lobular unit involution in benign tissues from Chinese breast cancer patients with luminal and triple-negative tumors. Breast Cancer Res 19: 61, 2017.

6. Zuo TT, Zheng RS, Zeng HM, Zhang SW and Chen WQ: Female breast cancer incidence and mortality in China, 2013. Thorac Cancer 8: 214-218, 2017.

7. McKevitt EC, Dingee CK, Leung SP, Brown CJ, Van Laeken NY, Lee $\mathrm{R}$ and Kuusk U: Reduced time to breast cancer diagnosis with coordination of radiological and clinical care. Cureus 9: e1919, 2017.

8. Nassar FJ, Nasr R and Talhouk R: MicroRNAs as biomarkers for early breast cancer diagnosis, prognosis and therapy prediction. Pharmacol Ther 172: 34-49, 2017.

9. Nagashima T, Sakakibara M, Sangai T, Kazama T, Nakatani Y and Miyazaki M: Tumor reduction rate predicts early recurrence in patients with breast cancer failing to achieve complete response to primary chemotherapy. Breast Cancer 17: 125-130, 2010.

10. Chu W, Jin W, Liu D, Wang J, Geng C, Chen L and Huang X: Diffusion-weighted imaging in identifying breast cancer pathological response to neoadjuvant chemotherapy: A meta-analysis. Oncotarget 9: 7088-7100, 2018

11. Cunningham D, Allum WH, Stenning SP, Thompson JN, Van de Velde CJ, Nicolson M, Scarffe JH, Lofts FJ, Falk SJ, Iveson TJ, et al: Perioperative chemotherapy versus surgery alone for resectable gastroesophageal cancer. N Engl J Med 355: 11-20, 2006.

12. Galli G, Bregni G, Cavalieri S, Porcu L, Baili P, Hade A, Di Salvo F, Sant M, Agresti R, Gennaro M, et al: Neoadjuvant chemotherapy exerts selection pressure towards luminal phenotype breast cancer. Breast care (Basel) 12: 391-394, 2017.

13. Alekseev NP, Vladimir II and Nadezhda TE: Pathological postpartum breast engorgement: Prediction, prevention, and resolution. Breastfeed Med 10: 203-208, 2015.

14. Amioka A, Masumoto N, Gouda N, Kajitani K, Shigematsu H, Emi A, Kadoya T and Okada M: Ability of contrast-enhanced ultrasonography to determine clinical responses of breast cancer to neoadjuvant chemotherapy. Jpn J Clin Oncol 46: 303-309, 2016.

15. Charehbili A, Wasser MN, Smit VT, Putter $\mathrm{H}$, van Leeuwen-Stok AE, Meershoek-Klein Kranenbarg WM, Liefers GJ, van de Velde CJ, Nortier JW and Kroep JR: Accuracy of MRI for treatment response assessment after taxane- and anthracycline-based neoadjuvant chemotherapy in HER2-negative breast cancer. Eur J Surg Oncol 40: 1216-1221, 2014.

16. Singh G, Kumar P, Parshad R, Seith A, Thulkar S and Hosten N: Role of color Doppler indices in predicting disease-free survival of breast cancer patients during neoadjuvant chemotherapy. Eur J Radiol 75: e158-e162, 2010.

17. Yang T, Zhang Z, Liu G, Mu D, Sun X, Chen Z, Liu Y, Wang C, Sun X and Wang Y: Accuracy of MRI for estimating residual tumor size after neoadjuvant chemotherapy in breast cancer with three-dimensional reconstruction technique. Zhonghua Wai Ke Za Zhi 53: 280-284, 2015 (In Chinese).

18. Baghbani F, Chegeni M, Moztarzadeh F, Mohandesi JA and Mokhtari-Dizaji M: Ultrasonic nanotherapy of breast cancer using novel ultrasound-responsive alginate-shelled perfluorohexane nanodroplets: In vitro and in vivo evaluation. Mater Sci Eng C Mater Biol Appl 77: 698-707, 2017.

19. Falou O, Sadeghi-Naini A, Prematilake S, Sofroni E, Papanicolau N, Iradji S, Jahedmotlagh Z, Lemon-Wong S, Pignol JP, Rakovitch E, et al: Evaluation of neoadjuvant chemotherapy response in women with locally advanced breast cancer using ultrasound elastography. Transl Oncol 6: 17-24, 2013. 
20. Garnier M, Champeaux E, Laurent E, Boehm A, Briard O, Wachter T, Vaillant L, Patat F, Bens G and Machet L: High-frequency ultrasound quantification of acute radiation dermatitis: Pilot study of patients undergoing radiotherapy for breast cancer. Skin Res Technol 23: 602-606, 2017.

21. Keune JD, Jeffe DB, Schootman M, Hoffman A, Gillanders WE and Aft RL: Accuracy of ultrasonography and mammography in predicting pathologic response after neoadjuvant chemotherapy for breast cancer. Am J Surg 199: 477-484, 2010.

22. Ma Y, Zhang S, Li J, Li J, Kang Y and Ren W: Comparison of strain and shear-wave ultrasounic elastography in predicting the pathological response to neoadjuvant chemotherapy in breast cancers. Eur Radiol 27: 2282-2291, 2017.

23. Itoh A, Ueno E, Tohno E, Kamma H, Takahashi H, Shiina T, Yamakawa $M$ and Matsumura T: Breast disease: Clinical application of US elastography for diagnosis. Radiology 239: 341-350, 2006

24. Duffaud F and Therasse P: New guidelines to evaluate the response to treatment in solid tumors. Bull Cancer 87: 881-886, 2000 (In French).

25. Eisenhauer EA, Therasse P, Bogaerts J, Schwartz LH, Sargent D, Ford R, Dancey J, Arbuck S, Gwyther S, Mooney M, et al: New response evaluation criteria in solid tumours: Revised RECIST guideline (version 1.1). Eur J Cancer 45: 228-247, 2009.

26. Baulies S, Belin L, Mallon P, Senechal C, Pierga JY, Cottu P, Sablin MP, Sastre X, Asselain B, Rouzier R and Reyal F: Time-varying effect and long-term survival analysis in breast cancer patients treated with neoadjuvant chemotherapy. $\mathrm{Br}$ J Cancer 113: 30-36, 2015.

27. Kern P, Von Minckwitz G, Puetter C, Pavlidou S, Flach A, Kimmig R and Rezai M: Prognostic impact of residual disease after neoadjuvant chemotherapy in 648 patients with triple-negative breast cancer. Anticancer Res 35: 5479-5484, 2015.

28. Miyoshi T, Shiobara M, Wakatsuki K, Arai S, Suda K, Miyazawa K, Aida T, Takahashi Y and Yoshioka S: Four cases of occult breast cancer treated with breast conserving therapy after neoadjuvant chemotherapy. Gan To Kagaku Ryoho 44: 1149-1151, 2017 (In Japanese).

29. Ohno N, Ishida M, Ohnishi K, Sawai K, Tabata S, Deguchi M, Kitajima T and Kaizaki Y: A case of pathological complete response following neoadjuvant chemotherapy with gemcitabine plus nab-paclitaxel in borderline resectable pancreatic cancer Gan To Kagaku Ryoho 44: 1735-1738, 2017 (In Japanese).

30. Lee SC, Grant E, Sheth P, Garcia AA, Desai B, Ji L, Groshen S, Hwang D, Yamashita M and Hovanessian-Larsen L: Accuracy of contrast-enhanced ultrasound compared with magnetic resonance imaging in assessing the tumor response after neoadjuvant chemotherapy for breast cancer. J Ultrasound Med 36: 901-911, 2017.

31. Zhou J, Luo Y, Ma BY,Ling WW and Zhu XL: Contrast-enhanced ultrasound diagnosis of hepatic metastasis of concurrent medullary-papillary thyroid carcinoma: A case report. Medicine (Baltimore) 96: e9065, 2017.
32. Sasada S, Masumoto N, Goda N, Kajitani K, Emi A, Kadoya T and Okada M: Dedicated breast PET for detecting residual disease after neoadjuvant chemotherapy in operable breast cancer: A prospective cohort study. Eur J Surg Oncol 44: 444-448, 2018.

33. Weber JJ, Jochelson MS, Eaton A, Zabor EC, Barrio AV Gemignani ML, Pilewskie M, Van Zee KJ, Morrow M and El-Tamer M: MRI and prediction of pathologic complete response in the breast and Axilla after neoadjuvant chemotherapy for breast cancer. J Am Coll Surg 225: 740-746, 2017.

34. Girotra M, Soota K, Dhaliwal AS, Abraham RR, Garcia-Saenz-de-Sicilia M and Tharian B: Utility of endoscopic ultrasound and endoscopy in diagnosis and management of hepatocellular carcinoma and its complications: What does endoscopic ultrasonography offer above and beyond conventional cross-sectional imaging? World J Gastrointest Endosc 10: 56-68, 2018.

35. Jia WR, Tang L, Wang DB, Chai WM, Fei XC, He JR, Chen M and Wang WP: Three-dimensional contrast-enhanced ultrasound in response assessment for breast cancer: A comparison with dynamic contrast-enhanced magnetic resonance imaging and pathology. Sci Rep 6: 33832, 2016.

36. Xu HD and Zhang YQ: Evaluation of the efficacy of neoadjuvant chemotherapy for breast cancer using diffusion-weighted imaging and dynamic contrast-enhanced magnetic resonance imaging. Neoplasma 64: 430-436, 2017.

37. Dietrich CF, Averkiou M, Nielsen MB, Barr RG, Burns PN, Calliada F, Cantisani V, Choi B, Chammas MC, Clevert DA, et al: How to perform Contrast-Enhanced Ultrasound (CEUS). Ultrasound Int Open 4: E2-E15, 2018.

38. Ang J, Hu L, Huang PT, Wu JX, Huang LN, Cao CH, Zheng YX and Chen L: Contrast-enhanced ultrasonography assessment of gastric cancer response to neoadjuvant chemotherapy. World J Gastroenterol 18: 7026-7032, 2012.

39. Kitamura H, Koike S, Nakazawa K, Matsumura H, Yokoi K, Nakagawa K and Arai M: A reversal in the vascularity of metastatic liver tumors from colorectal cancer after the cessation of chemotherapy plus bevacizumab: Contrast-enhanced ultrasonography and histological examination. J Surg Oncol 107: 155-159, 2013.

40. Yerli H, Yilmaz T, Kaskati T and Gulay H: Qualitative and semiquantitative evaluations of solid breast lesions by sonoelastography. J Ultrasound Med 30: 179-186, 2011.

41. Zhu Y, Deng S, Zhang Y and Jiang Q: Comparative study of ultrasonic elastography and conventional ultrasound in diagnosis of malignant anus neoplasm. Exp Ther Med 15: 2343-2346, 2018.

This work is licensed under a Creative Commons Attribution-NonCommercial-NoDerivatives 4.0 International (CC BY-NC-ND 4.0) License. 